\title{
Severity of Neuropsychiatric Symptoms in Nursing Home Residents
}

\author{
Anne-Sofie Helvik ${ }^{a-c}$ Knut Engedalc Bei Wu ${ }^{\text {h, }}{ }^{c}$ Jūrate Šaltytè Benth ${ }^{d, f, g}$ \\ Kirsten Corazzini $^{\text {h }}$ Irene Røen ${ }^{g}$ Geir Selbæk ${ }^{c, e, g}$ \\ ${ }^{a}$ Department of Public Health and General Practice, Faculty of Medicine, Norwegian

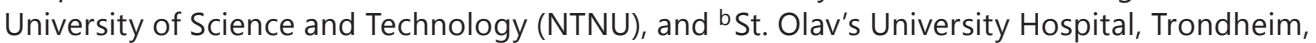 \\ 'Norwegian National Advisory Unit on Aging and Health, Vestfold Health Trust, Tønsberg, \\ ${ }^{d}$ Institute of Clinical Medicine, Campus Ahus, and Institute of Health and Society, Faculty of \\ Medicine, University of Oslo, Oslo, ${ }^{\mathrm{f}} \mathrm{H} \varnothing \mathrm{KH}$, Research Centre, Akershus University Hospital, \\ Lørenskog, and ${ }^{9}$ Centre for Old Age Psychiatric Research, Innlandet Hospital Trust, Ottestad, \\ Norway; ${ }^{\text {h}}$ Duke Center for the Study of Aging and Human Development, Duke University \\ School of Nursing, and 'Duke Global Health Institute, Durham, N.C., USA
}

\section{Key Words}

Neuropsychiatric symptoms · Nursing home $\cdot$ Neuropsychiatric Inventory

Nursing Home Version

\section{Abstract}

We aimed at assessing time shift in the severity of neuropsychiatric symptoms (NPS) in nursing home residents between 2004/2005 and 2010/2011 and associations between NPS and socio-demographic variables, physical health status, dementia severity, and the use of psychotropic drugs. The Neuropsychiatric Inventory Nursing Home Version was used in 2004/2005 $(n=1,163)$ and 2010/2011 $(n=1,858)$. Linear mixed model analysis was applied. There was no time shift in the severity of apathy, psychosis, and affective symptoms, but agitation did exhibit a time shift. Agitation was less severe in 2010/2011 than in 2004/2005 in residents with a Clinical Dementia Rating (CDR) sum of boxes score $\leq 4$, and more severe in residents with a CDR sum of boxes score $>16$. Higher CDR sum of boxes scores and use of psychotropic medication were associated with more severe apathy, agitation, psychosis, and affective symptoms. Poor physical health was associated with more severe apathy, psychosis, and affective symptoms. Women had more severe agitation and less severe affective symptoms than men. A longer stay in a nursing home was associated with more severe agitation and less severe affective symptoms. In conclusion, agitation was less severe in 2010/2011 than in 2004/2005 among nursing home residents with a milder degree of dementia, and more severe in residents with severe dementia. 


\section{Introduction}

Dementia is common among aged populations $[1,2]$, and almost all people with dementia will experience some type of neuropsychiatric symptoms (NPS) during the course of a dementia disorder of any aetiology [3]. NPS, also called 'behavioural and psychological symptoms of dementia', include psychiatric symptoms such as delusions, hallucination, depression, anxiety, and euphoria, as well as behavioural symptoms such as agitation, aggression, apathy, and disinhibition.

NPS are linked to the underlying brain disease causing dementia, and the symptoms appear to be a consequence of multiple, interacting internal and external risk factors [4], i.e. individual factors (such as age, gender, marital status, physical functioning, and physical health) and environmental factors (such as the type and degree of assistance). The relationship between NPS and these factors, the disease itself (including the degree and type of dementia), and the use of psychotropic medication is not fully understood [4-8].

NPS contribute to a feeling of distress and discomfort in people with dementia and are associated with poorer quality of life $[9,10]$. Furthermore, these symptoms tend to increase depression and the burden to informal family and formal caregivers [11], and increase the cost of care [12]. NPS are considered one of the leading causes of institutionalization for people with dementia [13] and are among the most significant challenges in dementia care [4].

In recent years, person-centred care has been implemented in Norwegian nursing homes in order to increase the quality of dementia care and to prevent and reduce NPS [14]. The framework for person-centred care includes valuing people with dementia, providing individualized care, and understanding the world from the demented person's perspective and providing a social environment that supports the needs of the person with cognitive impairment [15]. Care is based on principles of human rights such as respect, dignity, and having genuine choices $[14,16,17]$.

However, psychotropic medications are currently one of the most commonly deployed management strategies. Such drugs often have sub-optimal risk-benefit profiles and may not have an impact on some of the most frequently occurring symptoms [4]. These drugs are associated with an increased risk of adverse events in people with dementia [18]. It is recommended that non-pharmacological therapies and behavioural management are the first-line treatment [19], but the evidence for such treatment is still uncertain [20].

In Norway, as in other Western countries, a large proportion of nursing home residents suffer from dementia [21-25], and NPS are highly prevalent among these residents [26]. A recent review based on 7 follow-up studies found the prevalence of NPS relatively unchanged during follow-up, but the persistence, incidence, and cumulative prevalence varied considerably between the studies [26]. The frequency and severity of most NPS are associated with more severe dementia, but the number of studies is limited and the results concerning affective symptoms in relation to the severity of dementia are inconclusive [5, 6, 26-29]. However, no studies have compared the severity of NPS in samples of nursing home residents over time in the same country $[5,6,26,27]$. Even if more studies from the same country or region exist, they may not have used the same methodology and assessment tools, which makes a direct comparison difficult. Thus, it is a moot question whether the severity of NPS in nursing home residents is stable over time. It is vital for policy makers and health planners to have access to comparable studies on the severity of NPS among nursing home residents over time, since such information may give national health care planners important tools to help organize care.

The aim of this study was to compare the severity of NPS sub-syndromes between two large cross-sectional samples of nursing home residents (2004/2005 and 2010/2011) in 
order to assess a possible time shift. We further aimed to explore associations between NPS and socio-demographic variables, physical health status, functioning in activities of daily living, the severity of dementia, the use of psychotropic drugs, and the type of care unit.

\section{Subjects and Methods}

\section{Design}

The study included data from two cross-sectional samples of nursing home residents in Norway. The assessment of the first sample took place between November 2004 and January 2005 [30], while the assessment of the second sample took place between June 2010 and November 2011.

\section{Setting and Participants}

In total, Norway has 40,000 nursing home places (beds) [31] for a population of about 5 million, with about $14 \%(700,000)$ aged 65 years or older [32]. The jurisdiction for public health care services lies within the local municipalities, and the local authorities offer social services (such as housing and home services), in-home nursing and institutional care (mainly in nursing homes), and both long- and short-term care and rehabilitation.

Residents with a stay of at least 14 days were eligible for inclusion in both samples [30]. In 2004/2005, residents in 26 nursing homes in 18 municipalities were approached. This was a selection made to reflect the distribution of small, medium, and large municipalities. In all, 1,165 residents were eligible for inclusion, and only 2 declined to participate. In 2010/2011, residents in 24 of the 26 nursing homes from the previous sample were approached again, in addition to residents in 40 other nursing homes from 31 municipalities. In all, 2,385 individuals were eligible for inclusion; 423 declined participation either personally or through their next of kin, 33 had a serious somatic disease or terminal condition, 17 died prior to assessment, 1 left the nursing home prior to assessment, and 53 were excluded without any specified reason. Thus, the number of participants in 2010/2011 was 1,858.

\section{Measures}

NPS were assessed using the Neuropsychiatric Inventory Nursing Home Version (NPI-NH) [33] in a translated and validated Norwegian version [34]. The 10-item inventory covers the following symptoms: delusion, hallucination, euphoria, agitation/aggression, disinhibition, irritability/lability, depression/dysphoria, anxiety, apathy/indifference, and aberrant motor behaviour (no/yes). For each symptom, severity (score 1-3) multiplied by frequency (score 1-4) provides a score from 0 to 12 . Based on a previous principal component analysis, psychosis (delusions, hallucination), agitation (agitation/aggression, disinhibition, irritability), and affective (depression, anxiety) sub-syndrome scores were formed by summing the scores of the included items [28, 35-37]. Apathy/indifference was analysed as a single symptom.

Dementia and severity of dementia were assessed using the Clinical Dementia Rating (CDR) scale, covering 6 domains (memory; orientation; judgment and problem solving; community affairs; home and hobbies, and personal care) with 5 response categories $(0,0.5$, $1,2$, or 3$)[38,39]$. The total score is calculated using an algorithm that gives priority to memory [38]. Residents with a total score of $\geq 1$ were regarded as having dementia. A cut-off CDR score of $\geq 1$ defining dementia has been found adequate in previous Norwegian and international studies [40-42]. The categorical scores indicate the severity of dementia: a CDR score of 1 represents mild dementia, a CDR score of 2 represents moderate dementia, and a CDR score of 3 represents severe dementia. The sum score of the 6 domains (sum of boxes), 
ranging from 0 to 18, can also be used to measure the severity of dementia, with a higher score indicating more severe dementia. The correlation between the categorical CDR score and the CDR sum of boxes score is high $(\geq 0.9)[43,44]$. The Spearman correlation between the categorical CDR score and the CDR sum of boxes score in the present study was 0.93 . Due to a wider range of values, the CDR sum of boxes offers important advantages when analysing the data [44].

In both studies, we used the CDR score (CDR score $\geq 1$ ) as an indication of dementia, as it was not possible to perform a standardized dementia workup for all residents because many of them were too frail or mentally impaired to take part in examinations such as CT or MRI. A large number of residents with a CDR score of 3 could not be tested with any dementia tests such as the Mini-Mental State Examination or the clock drawing test.

Psychotropic medications were grouped according to the ATC code into the following categories: antipsychotics (N05A except lithium), antidepressants (N06A), anxiolytics (N05B), hypnotics/sedatives (N05C), and anti-dementia medication (N06D) (yes/no) [45]. The information was collected from the medical record of each resident [30].

The Personal Activities of Daily Living (P-ADL) score was assessed with the Physical Self-Maintenance Scale, including 6 items, with a total score ranging from 6 to 30 [46]. High scores indicate a lower level of functioning.

Physical health was assessed using the General Medical Health Rating (GMHR) scale [47]. This is a 1-item, global rating scale with 4 categories: good, fairly good, poor, and very poor. The rating was based on all available information on physical health and use of drugs. The scale has been used in large studies including older people with and without dementia [48] and has been translated and used in Norway [49].

Demographic information such as age, gender, marital status, and length of stay in the nursing home at the time of inclusion in the study was collected from the medical records. The type of unit was also recorded from among the following options: regular unit (RU), special care unit for people with dementia (SCU), rehabilitation unit (REU), and other units (OU), mainly psychogeriatric wards. The length of stay in a nursing home before study inclusion was measured in days.

\section{Procedure}

Nurses with wide clinical experience collected the data from both samples. Prior to the data collection, all assessors participated in a 2-day course on how to apply the standardized questionnaires. The nurses collected the data from medical records and a standardized interview with the residents' primary caregivers, all of whom were registered nurses. A pilot study including 41 nursing home residents was carried out prior to the first data collection to assess the inter-rater reliability of the CDR. It was performed between 1 geriatric psychiatrist (GP) and 2 other assessors, a registered nurse (RN) and a nurse specialized in psychiatry (NP). The kappa statistics for the global CDR score were 1 (GP vs. NP) and 0.86 (GP vs. RN and NP vs. RN). More detailed information has been published elsewhere [30]. Information regarding the study was given to the residents and their family members. In 2004/2005, the residents and their next of kin were informed that they could refuse to participate at any stage of the study. In 2010/2011, informed consent was obtained. If a resident lacked the ability to consent, the resident's next of kin signed on behalf of the resident. These procedures were recommended and approved by the Regional Ethics Committee in the south east of Norway, the Data Inspectorate and the Directorate for Health and Social Affairs in 2004 and 2010.

Data Analysis

The data from the nursing homes in this study exhibit a hierarchical structure. Moreover, a proportion of the nursing homes $(24$ of $66 ; 36.4 \%)$ and some of the participants (90 of 
1,$163 ; 7.7 \%$ ) were included in both samples. Hence, a cluster effect might be present at both the nursing home and the participant level, and statistical methods correctly adjusting for such an effect are needed.

Demographic and clinical characteristics of the two study samples are presented as means and standard deviations (SD), or frequencies and percentages, as appropriate. Between the studies, characteristics were compared using the linear mixed model (SAS MIXED procedure) for continuous data; the logistic or multinomial regression model (SAS GLIMMIX procedure) was used to compare the categorical characteristics. Both types of model contained a fixed effect for characteristics and a random effect for nursing homes.

The outcomes (degree of apathy and agitation, as well as psychotic and affective subsyndromes of NPS) were assessed with a linear regression model for hierarchical data with random intercepts for both nursing homes and participants, with participants nested within nursing homes (SAS MIXED procedure). The modelling strategy was the same for all outcomes. Bivariate regression models with fixed effects for independent variables [year of assessment, age, gender, marital status, severity of dementia (CDR sum of boxes), P-ADL, physical health, use of psychotropic drugs, length of stay in nursing home at inclusion, and type of care unit] were estimated first. Next, multivariate models containing all independent variables as fixed effects were estimated. In addition, the interaction between severity of dementia and year of study was assessed for all outcomes. Interactions were left in the multivariate model only if relevant according to Akaike's information criterion. Only cases with no missing values on independent variables were used in the regression analyses.

The results of the regression analyses are presented as regression coefficients with the corresponding 95\% confidence intervals (CI), or standard errors (SE) in the case of a present interaction. To ease the interpretation of an interaction term, the results were tabulated as estimated means with 95\% CI and presented graphically. All analyses were performed in SPSS version 22 and SAS version 9.3. p values $<0.05$ were considered statically significant. All tests were two-sided.

\section{Results}

In total, 1,163 nursing home residents participated in 2004/2005, and 1,858 participated in $2010 / 2011$. As shown in table 1 , a simple comparison of nursing home residents between $2004 / 2005$ and $2010 / 2011$ showed that the participants in 2010/2011 were significantly older and used significantly fewer antipsychotics regularly than the nursing home residents in $2004 / 2005$.

\section{Apathy}

The mean (SD) apathy sub-syndrome score was 2.2 (3.7) in 2004/2005 and 2.0 (3.4) in $2010 / 2011$, respectively (table 2). There was no time shift in apathy according to the multivariate regression model (table 3). Lower age, higher CDR sum of boxes score, poorer P-ADL function (higher score), poorer physical health, use of antipsychotics, and use of antidepressants were associated with increased severity of apathy.

\section{Agitation}

The mean (SD) agitation sub-syndrome score of the residents was 5.8 (8.0) in 2004/2005 and 6.1 (8.3) in 2010/2011, respectively (table 2). There was a significant interaction between study time and CDR sum of boxes score in a multivariate linear mixed model (table 3). According to the model, the severity of agitation was significantly lower in 2010/2011 than in 2004/2005 among residents with a CDR sum of boxes score $\leq 4$. However, the agitation 
Table 1. Characteristics of the two study samples of residents in nursing homes
Table 2. Severity of NPS and sub-syndromes

\begin{tabular}{l|l}
\hline DOI: 10.1159/000442250 & $\begin{array}{l}\text { C) } 2016 \text { The Author(s). Published by S. Karger AG, Basel } \\
\text { www.karger.com/dee }\end{array}$ \\
\hline Helvik et al.: Severity of Neuropsychiatric Symptoms in Nursing Home Residents
\end{tabular}

\begin{tabular}{|c|c|c|c|}
\hline Variables & $\begin{array}{l}\text { Study I } \\
(2004 / 2005) \\
(n=1,163)\end{array}$ & $\begin{array}{l}\text { Study II } \\
(2010 / 2011) \\
(n=1,858)\end{array}$ & $\mathrm{p}$ value \\
\hline \multicolumn{4}{|l|}{ Demographics } \\
\hline Age, years & $84.4 \pm 7.8$ & $85.5 \pm 8.00$ & $0.004^{1}$ \\
\hline Women & $846(72.7)$ & $1,313(70.7)$ & $0.401^{2}$ \\
\hline Single as marital status & $941(81.0)$ & $1,426(77.5)$ & $0.086^{2}$ \\
\hline \multicolumn{4}{|l|}{ Dementia } \\
\hline None (CDR score <1) & 227 (19.6) & $286(15.7)$ & $0.103^{3}$ \\
\hline Mild (CDR score 1 ) & $232(20.0)$ & $311(17.1)$ & \\
\hline Moderate (CDR score 2) & $310(26.7)$ & $525(28.8)$ & \\
\hline Severe (CDR score 3) & $390(33.6)$ & $702(38.5)$ & \\
\hline CDR sum of boxes score & $11.2 \pm 5.3$ & $11.7 \pm 5.0$ & $0.323^{1}$ \\
\hline \multicolumn{4}{|l|}{ Information on health } \\
\hline P-ADL score & $18.1 \pm 5.4$ & $17.9 \pm 5.4$ & $0.726^{1}$ \\
\hline \multicolumn{4}{|l|}{ Physical health } \\
\hline Good & $194(17.2)$ & $283(15.3)$ & $0.184^{3}$ \\
\hline Fair & $386(34.1)$ & $724(39.2)$ & \\
\hline Poor & $378(33.4)$ & $664(36.0)$ & \\
\hline Very poor & $173(15.3)$ & $174(9.4)$ & \\
\hline \multicolumn{4}{|c|}{ Use of psychotropic medication } \\
\hline Antipsychotics & $280(24.1)$ & $311(16.7)$ & $0.001^{2}$ \\
\hline Antidepressants & $445(38.3)$ & $675(36.3)$ & $0.597^{2}$ \\
\hline Anxiolytics & $282(24.2)$ & 405 (21.8) & $0.672^{2}$ \\
\hline Sedatives & $337(29.0)$ & $563(30.3)$ & $0.695^{2}$ \\
\hline Anti-dementia drugs & $131(11.3)$ & $279(15.0)$ & $0.258^{2}$ \\
\hline \multicolumn{4}{|l|}{ Nursing home care } \\
\hline Length of stay ${ }^{4}$, days & $938.3 \pm 1,013.0$ & $948.5 \pm 1,046.7$ & $0.717^{1}$ \\
\hline \multicolumn{4}{|l|}{ Type of care unit } \\
\hline RU & $762(65.5)$ & $1,152(62.4)$ & $0.929^{3}$ \\
\hline SCU & 313 (26.9) & $502(27.2)$ & \\
\hline REU & $36(3.1)$ & $48(2.6)$ & \\
\hline $\mathrm{OU}$ & $52(4.5)$ & 145 (7.9) & \\
\hline
\end{tabular}

Values are expressed as means \pm SD or $\mathrm{n}(\%)$. Italics denote significance. ${ }^{1}$ Linear mixed model. ${ }^{2}$ Logistic regression model for hierarchical data.

${ }^{3}$ Multinomial regression model for hierarchical data. ${ }^{4} \mathrm{p}$ value calculated on ln scale.

\begin{tabular}{llllll}
\hline Variables & \multicolumn{2}{l}{ Study I $(2004 / 2005)$} & & \multicolumn{2}{l}{ Study II $(2010 / 2011)$} \\
\cline { 2 - 3 } \cline { 6 - 7 } & $\mathrm{n}$ & score $^{1}$ & & & \multicolumn{2}{c}{ score $^{1}$} \\
\hline Apathy & 1,160 & $2.2 \pm 3.7$ & & 1,852 & $2.0 \pm 3.4$ \\
Agitation & 1,157 & $5.8 \pm 8.0$ & & 1,847 & $6.1 \pm 8.3$ \\
Psychosis & 1,159 & $2.8 \pm 5.1$ & & 1,852 & $2.7 \pm 5.1$ \\
Affective symptoms & 1,158 & $3.6 \pm 5.3$ & & 1,851 & $3.4 \pm 5.1$
\end{tabular}

Scores are expressed as means \pm SD. ${ }^{1}$ Unadjusted for cluster effect. 
Dementia

and Geriatric

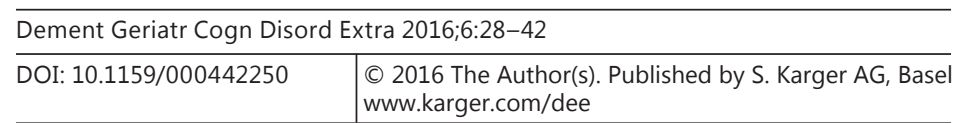

Helvik et al.: Severity of Neuropsychiatric Symptoms in Nursing Home Residents

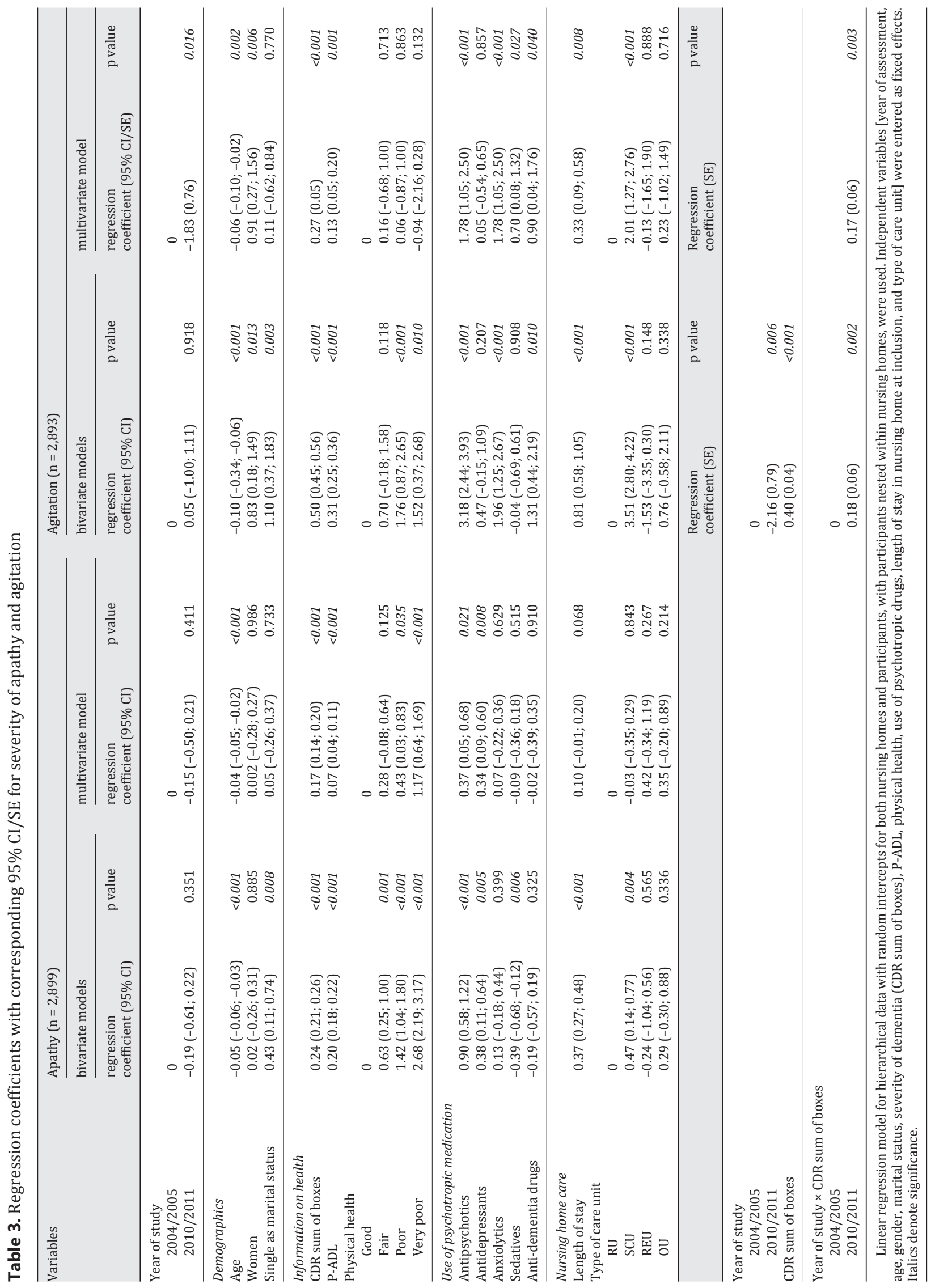


Table 4. Means (95\% CI) from the multivariate linear mixed model for agitation (table 3) estimated for different CDR sum of boxes scores

\begin{tabular}{rllll}
\hline CDR score & 2004 & 2011 & Difference $2004-2011$ & p value \\
\hline 2 & $2.24(-1.53 ; 6.02)$ & $0.75(-3.00 ; 4.50)$ & $1.49(0.20 ; 2.79)$ & 0.024 \\
4 & $2.79(-0.99 ; 6.56)$ & $1.63(-2.14 ; 5.40)$ & $1.16(0.03 ; 2.28)$ & 0.044 \\
6 & $3.33(-0.46 ; 7.12)$ & $2.51(-1.27 ; 6.30)$ & $0.82(-0.16 ; 1.79)$ & 0.100 \\
8 & $3.87(0.06 ; 7.69)$ & $3.40(-0.42 ; 7.21)$ & $0.48(-0.38 ; 1.33)$ & 0.273 \\
10 & $4.42(0.57 ; 8.26)$ & $4.28(0.43 ; 8.13)$ & $0.14(-0.64 ; 0.92)$ & 0.728 \\
12 & $4.96(1.07 ; 8.85)$ & $5.16(1.27 ; 9.06)$ & $-0.20(-0.97 ; 0.57)$ & 0.611 \\
14 & $5.51(1.57 ; 9.45)$ & $6.04(2.10 ; 9.99)$ & $-0.54(-1.36 ; 0.28)$ & 0.198 \\
16 & $6.05(2.05 ; 10.05)$ & $6.93(2.92 ; 10.93)$ & $-0.88(-1.80 ; 0.05)$ & 0.063 \\
18 & $6.59(2.52 ; 10.66)$ & $7.81(3.74 ; 11.88)$ & $-1.22(-2.28 ;-0.15)$ & 0.025 \\
\hline
\end{tabular}

Italics denote significance.

Fig. 1. Means and 95\% CI for agitation in 2004/2005 and 2010/2011 for different CDR sum of boxes scores.

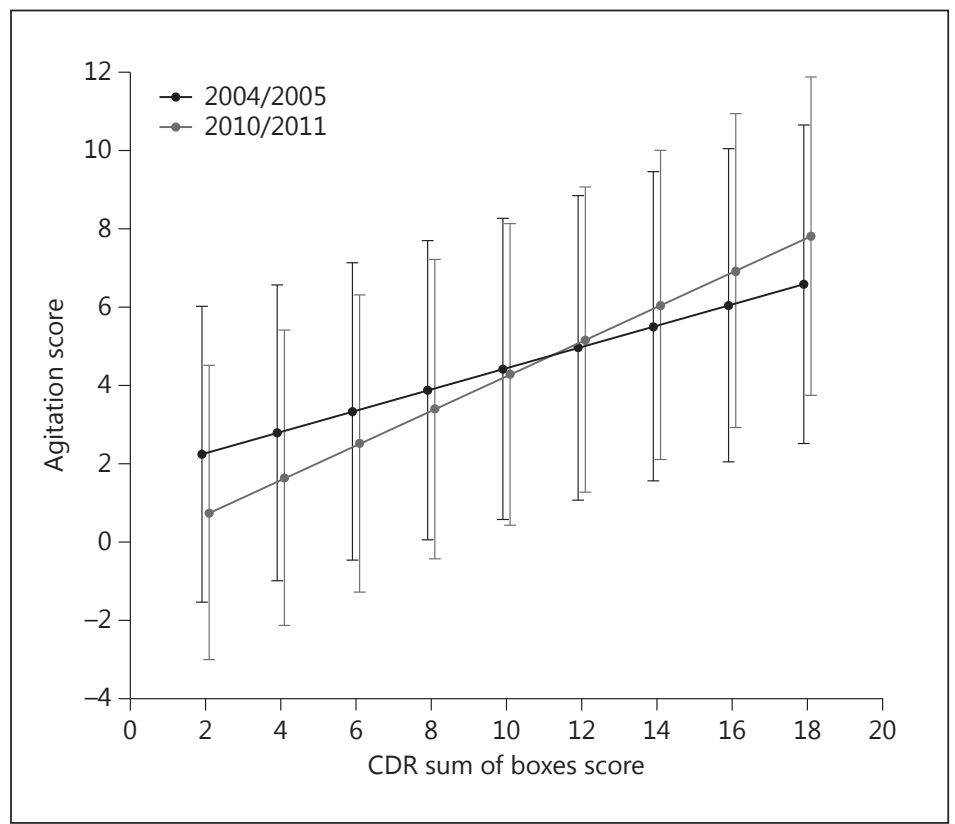

level was significantly higher in 2010/2011 than in 2004/2005 among residents with a CDR sum of boxes score $>16$. For more details, see table 4 and figure 1 . Lower age, female gender, poorer P-ADL function (higher score), use of antipsychotics, anxiolytics, sedatives, and antidementia medication as well as longer stay in a nursing home were associated with more severe agitation. The agitation was more severe in SCU than in RU in the nursing homes.

\section{Psychosis}

The mean (SD) psychosis sub-syndrome score of the residents was 2.8 (5.1) in 2004/2005 and 2.7 (5.1) in 2010/2011, respectively (table 2). There was no time shift in the severity of psychosis according to the multivariate regression analysis (table 5). Higher CDR sum of boxes score, poor physical health, and use of antipsychotics, anxiolytics, and anti-dementia drugs were significantly associated with increased severity of psychosis. The severity of 


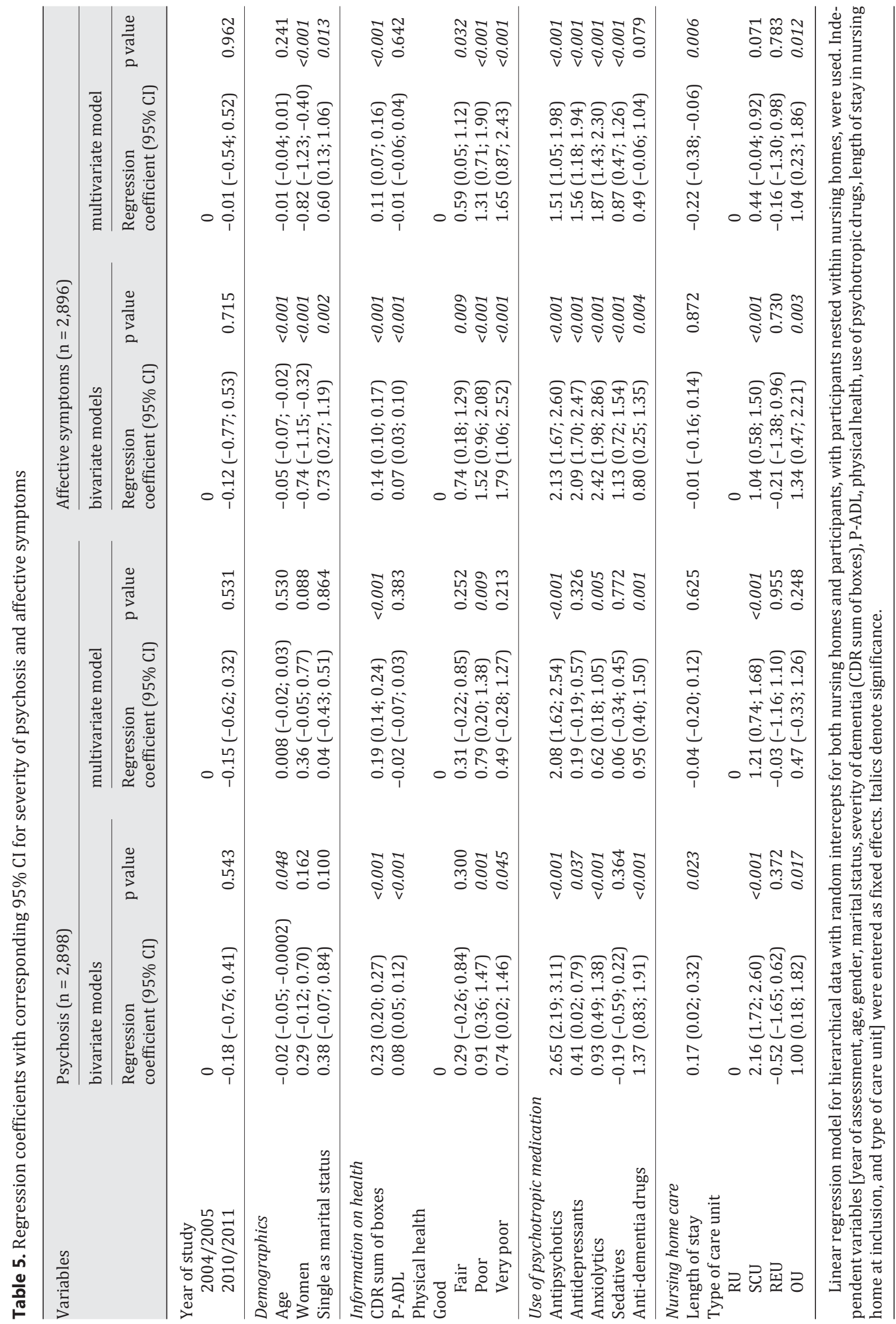


psychosis was higher among residents living in SCU than among those living in RU in the nursing homes.

\section{Affective Symptoms}

The mean (SD) affective sub-syndrome score of the residents was 3.6 (5.3) in 2004/2005 and 3.4 (5.1) in 2010/2011, respectively (table 2). No time shift was present in the severity of the affective sub-syndrome (table 5). Being a man, being single, a higher CDR sum of boxes score, poorer physical health, a shorter nursing home stay, as well as the use of antipsychotics, antidepressants, anxiolytics, and sedatives were associated with increased severity of the affective sub-syndrome. In addition, the severity of the affective sub-syndrome was significantly higher in OU than in RU.

\section{Discussion}

NPS were compared between residents from two nursing home studies conducted in the same country during a 6-year interval. There was no time shift in the severity of apathy, psychosis, and affective symptoms, but the severity of agitation was significantly lower in 2010/2011 than in 2004/2005 for residents with CDR sum of boxes scores $\leq 4$, and significantly worse in residents with the highest CDR sum of boxes scores ( $>16)$. Residents in SCU had more severe agitation and psychosis than residents in RU. A longer duration of stay in a nursing home was associated with more severe agitation and less severe affective symptoms. In addition, more severe dementia, use of psychotropic medication, and somatic health difficulties were associated with more severe NPS. Women had more severe symptoms of agitation and less severe affective symptoms than men.

The environment is known to influence the presence and severity of NPS, including the psychosocial environment and the care given $[4,6]$. The present study found that the severity of NPS in two different nursing home samples was quite stable over time, but there was a time shift in the severity of agitation. Even if the Norwegian nursing homes did not undergo any major organizational changes in the follow-up period, it is possible that those with lower CDR scores in 2010/2011 received care which was better adjusted to their needs than did those in 2004/2005. This effect may partially be a result of the introduction of person-centred care in Norwegian nursing homes in the 6-year period $[15,50]$. Even if there is an ongoing system change in Norway, we do not have detailed information about the philosophy of nursing practice and person-centred care at the institutions participating in the present study. Stress and strain related to daily routines such as bathing, dressing, and toileting may cause frustration and agitation in patients with limited resources, and person-centred care may reduce such stress and strain $[16,17]$. However, this explanation cannot be the only one, because this would imply that the severity of agitation was lower in all residents in 2010/2011 compared to the residents in 2004/2005. Over the past few years, there has been an increased focus on the prescription of psychotropic medication and, particularly, the use of antipsychotic drugs in people with dementia. The change in prevalence of antipsychotic drug use from 2004/2005 (24.1\%) to $2010 / 2011$ (16.1\%) may also have an important impact on the time shift in agitation. However, this change does not seem to have benefited those with the most severe symptoms. Even though many residents with dementia can have their antipsychotic drugs discontinued without symptom exacerbation, previous studies have indicated that increasing symptoms may be present among those with the most severe symptoms [51].

SCU for patients with dementia exist within nursing homes. The intention is that a physical environment and care adapted to their disease contribute to reducing the stress and strain that people with dementia experience. In this study, access to SCU was offered to around 25\% 
of the residents, and the coverage did not increase during the 6-year period. However, more than half of the residents had moderate or severe dementia. We found that the severity of agitation and psychosis was higher among residents in SCU. The severity of NPS in these residents is most probably the reason for having offered them a stay in SCU, an explanation that conforms to our clinical experience. Given the nature of this study, however, we cannot rule out the possibility that being in an SCU is an additional, exacerbating factor for the severity of agitation and psychosis.

The duration of stay in a nursing home may influence the prevalence and severity of NPS. Independent of dementia severity, studies have noted that relocation to a nursing home per se increases the risk of affective symptoms [52,53]. Losses in life and the need to adjust to a new life situation are reasonable explanatory factors for the prevalence [54] and severity of affective symptoms. After some time, the affective symptoms may be attenuated due to residents learning to accommodate to the situation. We also found that residents with a longer nursing home stay had more severe agitation [28]. The nursing home environment is known to be a significant factor in increased agitation [6]. Residents spend a considerable number of daytime hours together in dining and living rooms, so their symptoms may become more severe over time due to the influence of behaviours of other residents with NPS [6]. Future research using approaches such as observational methodologies to study if and how the environmental settings over time can affect NPS would provide explanatory data to help interpret such relationships.

Even if care- and environment-related issues are important in nursing homes studies, factors related to the disease, the use of psychotropic medication, physical health, and demographic conditions must be considered to understand the severity of NPS. The cross-sectional design of the present study showed that residents with higher CDR sum of boxes scores had more severe apathy, agitation, psychosis, and affective symptoms. In line with our findings, a recent 53-month follow-up study of nursing home residents with dementia found more severe dementia at one time point to be associated with more severe apathy, agitation, and psychosis at the same time point [28]. There is as yet no clear consensus on the association between severity of dementia symptoms and severity of affective symptoms [6, 26-29], even if NPS generally tend to worsen when the severity of dementia symptoms increases $[5,6,26]$. Thus, the association found between increasing CDR sum of boxes scores and more severe affective symptoms should be interpreted with caution. It has repeatedly been found that the use of psychotropic medication may not have the expected effect on NPS [4] and could have considerable adverse effects [18]. In the present study, use of such medication was associated with a higher severity of NPS. Due to the nature of the study, we do not know whether this is because of lacking or adverse effects of the medication. It could also be that people taking psychotropic medication had had even more severe symptoms prior to drug initiation. Poor physical health, measured by general medical health and P-ADL, was associated with worse NPS in the present study. More pronounced ADL impairment has been linked with NPS in several studies, and in some studies, poorer physical health has been found to be related to a lower prevalence of aggression, but those results are conflicting [6]. Pain, which was not assessed in the present study, may be a confounder. A recent review of 18 studies found pain to be weakly associated with both physical functioning and NPS [55].

In the present study, we found that women displayed a lower severity of affective symptoms and more severe agitation than men. Some previous nursing home studies have found depression to be more prevalent in men [56] and agitation to be more prevalent in women $[7,50,57]$, but the results are conflicting $[6,58]$. The importance of gender for the severity of NPS is less studied than its importance for the prevalence of NPS, and the impact of gender as a basic biologic variable in relation to dementia and dementia-related challenges remains elusive [59]. Also, the impact of age on NPS is inconclusive [6]. It has been reported 
that aggression $[56,57]$ and restlessness $[60]$ are more prevalent in younger nursing home residents, while other studies have not found a relationship between age and the prevalence of such symptoms [6]. We found that the younger residents had more severe agitation and apathy.

Our study has significant strengths. Firstly, all nurses participating in data collection took part in a 2-day educational course to secure adequate knowledge and a high data quality prior to collecting the data for this study. Secondly, a large sample size at both time points enabled us to adjust for many potentially important variables, such as health and demographic factors. Thirdly, this study benefits from the inclusion of nursing homes located in a large part of the country. However, we cannot guarantee that the sample is representative of Norwegian nursing home residents, since inclusion was not based on random selection from all nursing homes in Norway. Finally, additional nursing homes from new municipalities were added to the study at the second time point, but statistical analysis was adjusted for a possible cluster effect within nursing homes. Also, a small proportion of the residents participated in the study twice, which was adjusted for in the statistical analyses.

The study has some limitations. Firstly, we did not apply the exact same inclusion procedure at the second collection time point, but we do not consider it likely that we systematically biased the resident sample through a higher proportion of withdrawals of residents in $2010 / 2011$. At this time point, new research regulations in Norway required informed consent - or, in cases where residents lacked the ability to consent, the residents' next of kin had to sign on behalf of the residents before a professional primary care taker could give out resident information [61]. Secondly, it is a limitation that the assessments of dementia and the degree of dementia are based on the CDR evaluation of several assessors, and that a CDR assessment was used and not a standardized dementia diagnosis, including neuropsychological tests. However, the CDR is an accepted assessment tool and commonly used in epidemiological nursing home studies to identify dementia and measure the severity of dementia $[43,62]$, and the agreement between the CDR and a diagnostic procedure by the ICD-10 is high [40]. Lastly, we would like to point out that pain, which can be a confounder, was not assessed and that no conclusions about causality can be drawn due to the cross-sectional nature of the study.

\section{Conclusion}

The severity of apathy, psychosis, and affective symptoms did not change between $2004 / 2005$ and 2010/2011. Agitation was less severe in 2010/2011 than in 2004/2005 among nursing home residents with milder degrees of dementia, and more severe in residents with very severe degrees of dementia.

\section{Acknowledgement}

Data collection was funded by unrestricted grants from the Eastern Norway Regional Health authorities.

\section{Disclosure Statement}

The authors declare that they have no competing interests. 


\section{References}

1 Ferri CP, et al: Global prevalence of dementia: a Delphi consensus study. Lancet 2005;366:2112-2117.

$>2$ Berr C, Wancata J, Ritchie K: Prevalence of dementia in the elderly in Europe. Eur Neuropsychopharmacol 2005;15:463-471.

-3 Steinberg M, et al: Point and 5-year period prevalence of neuropsychiatric symptoms in dementia: the Cache County Study. Int J Geriatr Psychiatry 2008;23:170-177.

4 Kales HC, Gitlin LN, Lyketsos CG: Management of neuropsychiatric symptoms of dementia in clinical settings: recommendations from a multidisciplinary expert panel. J Am Geriatr Soc 2014;62:762-769.

-5 Cummings JL, McPherson S: Neuropsychiatric assessment of Alzheimer's disease and related dementias. Aging (Milano) 2001;13:240-246.

6 Zuidema S, Koopmans R, Verhey F: Prevalence and predictors of neuropsychiatric symptoms in cognitively impaired nursing home patients. J Geriatr Psychiatry Neurol 2007;20:41-49.

$\rightarrow 7$ Zuidema SU, de Jonghe JF, Verhey FR, Koopmans RT: Predictors of neuropsychiatric symptoms in nursing home patients: influence of gender and dementia severity. Int J Geriatr Psychiatry 2009;24:1079-1086.

-8 Zuidema SU, de Jonghe JF, Verhey FR, Koopmans RT: Environmental correlates of neuropsychiatric symptoms in nursing home patients with dementia. Int J Geriatr Psychiatry 2010;25:14-22.

-9 Wetzels RB, Zuidema SU, de Jonghe JF, Verhey FR, Koopmans RT: Determinants of quality of life in nursing home residents with dementia. Dement Geriatr Cogn Disord 2010;29:189-197.

10 van de Ven-Vakhteeva J, Bor H, Wetzels RB, Koopmans RT, Zuidema SU: The impact of antipsychotics and neuropsychiatric symptoms on the quality of life of people with dementia living in nursing homes. Int J Geriatr Psychiatry 2013;28:530-538.

11 Sink KM, Covinsky KE, Barnes DE, Newcomer RJ, Yaffe K: Caregiver characteristics are associated with neuropsychiatric symptoms of dementia. J Am Geriatr Soc 2006;54:796-803.

12 Murman DL, Chen Q, Powell MC, Kuo SB, Bradley CJ, Colenda CC: The incremental direct costs associated with behavioral symptoms in AD. Neurology 2002;59:1721-1729.

13 Gaugler JE, Yu F, Krichbaum K, Wyman JF: Predictors of nursing home admission for persons with dementia. Med Care 2009;47:191-198.

$\$ 14$ Love K, Femia E: Helping individuals with dementia live more fully through person-centered practices. J Gerontol Nurs 2015;41:9-14.

-15 Rokstad AM, Røsvik J, Kirkevold O, Selbæk G, Šaltytė Benth J, Engedal K: The effect of person-centred dementia care to prevent agitation and other neuropsychiatric symptoms and enhance quality of life in nursing home patients: a 10-month randomized controlled trial. Dement Geriatr Cogn Disord 2013;36:340-353.

16 Sloane PD, et al: Effect of person-centered showering and the towel bath on bathing-associated aggression, agitation, and discomfort in nursing home residents with dementia: a randomized, controlled trial. J Am Geriatr Soc 2004;52:1795-1804.

17 Hall GR, Buckwalter KC: Progressively lowered stress threshold: a conceptual model for care of adults with Alzheimer's disease. Arch Psychiatr Nurs 1987;1:399-406.

18 Ballard C, Corbett A: Management of neuropsychiatric symptoms in people with dementia. CNS Drugs 2010; 24:729-739.

19 National Institute for Health and Clinical Excellence: Dementia: the NICE-SCIE Guideline on Supporting People with Dementia and Their Careers in Health and Social Care. National Clinical Practice Guideline No 42. Leister, British Psychological Society and Gaskell, 2007.

20 Ballard CG, et al: Management of agitation and aggression associated with Alzheimer disease. Nat Rev Neurol 2009;5:245-255.

-21 Seitz D, Purandare N, Conn D: Prevalence of psychiatric disorders among older adults in long-term care homes: a systematic review. Int Psychogeriatr 2010;22:1025-1039.

22 Schäufele M, Köhler L, Hendlmeier I, Hoell A, Weyerer S: Prevalence of dementia and medical care in German nursing homes: a nationally representative survey (in German). Psychiatr Prax 2013;40:200-206.

-23 Kowalska J, Rymaszewska J, Szczepanska-Gieracha J: Occurrence of cognitive impairment and depressive symptoms among the elderly in a nursing home facility. Adv Clin Exp Med 2013;22:111-117.

24 Bergh S, Engedal K, Røen I, Selbæk G: The course of neuropsychiatric symptoms in patients with dementia in Norwegian nursing homes. Int Psychogeriatr 2011;23:1231-1239.

-25 Bergh S, Holmen J, Saltvedt I, Tambs K, Selbæk G: Dementia and neuropsychiatric symptoms in nursing-home patients in Nord-Trøndelag County (in English, Norwegian). Tidsskr Nor Laegeforen 2012;132:1956-1959.

-26 Selbæk G, Engedal K, Bergh S: The prevalence and course of neuropsychiatric symptoms in nursing home patients with dementia: a systematic review. J Am Med Dir Assoc 2013;14:161-169.

27 Wetzels R, Zuidema S, Jansen I, Verhey F, Koopmans R: Course of neuropsychiatric symptoms in residents with dementia in long-term care institutions: a systematic review. Int Psychogeriatr 2010;22:1040-1053.

28 Selbæk G, Engedal K, Benth JŠ, Bergh S: The course of neuropsychiatric symptoms in nursing-home patients with dementia over a 53-month follow-up period. Int Psychogeriatr 2014;26:81-91.

29 Borza T, Engedal K, Bergh S, Barca ML, Benth JŠ, Selbæk G: The course of depressive symptoms as measured by the Cornell Scale for Depression in Dementia over 74 months in 1,158 nursing home residents. J Affect Disord 2015;175:209-216. 
-30 Selbæk G, Kirkevold 0, Engedal K: The prevalence of psychiatric symptoms and behavioural disturbances and the use of psychotropic drugs in Norwegian nursing homes. Int J Geriatr Psychiatry 2007;22:843-849.

31 Finansdepartementet: Statsbudsjettet 2013. 2013. http://www.statsbudsjettet.no/Statsbudsjettet-2013/ Budsjettsporsmal/Bevilgningssporsmal/Hyre144/?all=true\&parti=h\%C3\%B8yreOslo.

-32 Boltz M, Resnick B, Chippendale T, Galvin J: Testing a family-centered intervention to promote functional and cognitive recovery in hospitalized older adults. J Am Geriatr Soc 2014;62:2398-2407.

33 Cummings JL: The Neuropsychiatric Inventory: assessing psychopathology in dementia patients. Neurology 1997;48:S10-S16.

34 Selbæk G, Kirkevold O, Sommer OH, Engedal K: The reliability and validity of the Norwegian version of the Neuropsychiatric Inventory, Nursing Home Version (NPI-NH). Int Psychogeriatr 2008;20:375-382.

-35 Colombo M, Vitali S, Cairati M, Vaccaro R, Andreoni G, Guaita A: Behavioral and psychotic symptoms of dementia (BPSD) improvements in a special care unit: a factor analysis. Arch Gerontol Geriatr 2007;44(suppl 1):113-120.

-36 Zuidema SU, de Jonghe JF, Verhey FR, Koopmans RT: Neuropsychiatric symptoms in nursing home patients: factor structure invariance of the Dutch nursing home version of the Neuropsychiatric Inventory in different stages of dementia. Dement Geriatr Cogn Disord 2007;24:169-176.

-37 Selbæk G, Engedal K: Stability of the factor structure of the Neuropsychiatric Inventory in a 31-month followup study of a large sample of nursing-home patients with dementia. Int Psychogeriatr 2012;24:62-73.

38 Hughes CP, Berg L, Danziger WL, Coben LA, Martin RL: A new clinical scale for the staging of dementia. Br J Psychiatry 1982;140:566-572.

39 Morris JC: The Clinical Dementia Rating (CDR): current version and scoring rules. Neurology 1993;43:24122414.

40 Engedal K, Haugen P: The prevalence of dementia in a sample of elderly Norwegians. Int J Geriatr Psychiatry 1993;8:565-570.

41 Nygaard HA, Naik M, Ruths S: Mental impairment in nursing home residents (in Norwegian). Tidsskr Nor Laegeforen 2000;120:3113-3116.

$\checkmark 42$ Waite L, et al: Informant-based staging of dementia using the clinical dementia rating. Alzheimer Dis Assoc Disord 1999;13:34-37.

43 Mjørud M, Kirkevold M, Røsvik J, Selbæk G, Engedal K: Variables associated to quality of life among nursing home patients with dementia. Aging Ment Health 2014;18:1013-1021.

44 O’Bryant SE, et al: Staging dementia using Clinical Dementia Rating Scale Sum of Boxes scores: a Texas Alzheimer's Research Consortium study. Arch Neurol 2008;65:1091-1095.

45 WHO Collaborating Centre for Drug Statistics Methodology: Guidelines for ATC classification and DDD assignment, 2015. Oslo, WHO Collaborating Centre for Drug Statistics Methodology, 2014.

-46 Lawton MP, Brody EM: Assessment of older people: self-maintaining and instrumental activities of daily living. Gerontologist 1969;9:179-186.

47 Lyketsos CG, et al: The General Medical Health Rating: a bedside global rating of medical comorbidity in patients with dementia. J Am Geriatr Soc 1999;47:487-491.

48 Lyketsos C, et al: Population-based study of medical comorbidity in early dementia and 'cognitive impairment, no dementia (CIND)': association with functional and cognitive impairment: the Cache County Study. Am J Geriatr Psychiatry 2004;13:656-664.

49 Sylliaas H, Selbæk G, Bergland A: Do behavioral disturbances predict falls among nursing home residents? Aging Clin Exp Res 2012;24:251-256.

50 Burgio LD, Butler FR, Roth DL, Hardin JM, Hsu CC, Ung K: Agitation in nursing home residents: the role of gender and social context. Int Psychogeriatr 2000;12:495-511.

-51 Ballard CG, et al: A 3-month, randomized, placebo-controlled, neuroleptic discontinuation study in 100 people with dementia: the neuropsychiatric inventory median cutoff is a predictor of clinical outcome.J Clin Psychiatry 2004;65:114-119.

-52 Anstey KJ, von Sanden C, Sargent-Cox K, Luszcz MA: Prevalence and risk factors for depression in a longitudinal, population-based study including individuals in the community and residential care. Am J Geriatr Psychiatry 2007;15:497-505.

53 Pot AM, Deeg DJ, Twisk JW, Beekman AT, Zarit SH: The longitudinal relationship between the use of long-term care and depressive symptoms in older adults. Gerontologist 2005;45:359-369.

-54 Iden KR, Engedal K, Hjorleifsson S, Ruths S: Prevalence of depression among recently admitted long-term care patients in Norwegian nursing homes: associations with diagnostic workup and use of antidepressants. Dement Geriatr Cogn Disord 2014;37:154-162.

55 van Dalen-Kok AH, Pieper MJ, de Waal MW, Lukas A, Husebo BS, Achterberg WP: Association between pain, neuropsychiatric symptoms, and physical function in dementia: a systematic review and meta-analysis. BMC Geriatr 2015;15:49.

56 Brodaty H, et al: Psychosis, depression and behavioural disturbances in Sydney nursing home residents: prevalence and predictors. Int J Geriatr Psychiatry 2001;16:504-512.

57 Majic T, Pluta JP, Mell T, Treusch Y, Gutzmann H, Rapp MA: Correlates of agitation and depression in nursing home residents with dementia. Int Psychogeriatr 2012;24:1779-1789.

58 Lövheim H, Sandman PO, Karlsson S, Gustafson Y: Sex differences in the prevalence of behavioral and psychological symptoms of dementia. Int Psychogeriatr 2009;21:469-475. 
59 Carter CL, Resnick EM, Mallampalli M, Kalbarczyk A: Sex and gender differences in Alzheimer's disease: recommendations for future research. J Womens Health (Larchmt) 2012;21:1018-1023.

60 Wagner AW, Teri L, Orr-Rainey N: Behavior problems of residents with dementia in special care units. Alzheimer Dis Assoc Disord 1995;9:121-127.

61 Menichetti J, Libreri C, Lozza E, Graffigna G: Giving patients a starring role in their own care: a bibliometric analysis of the on-going literature debate. Health Expect 2014, Epub ahead of print.

62 Leoutsakos JM, et al: Effects of general medical health on Alzheimer's progression: the Cache County Dementia Progression Study. Int Psychogeriatr 2012;24:1561-1570. 